\title{
Les formes variées et évolutives de l'activation en Belgique
}

Vanessa De Greef

\section{Q OpenEdition}

1 Journals

Édition électronique

URL : https://journals.openedition.org/rdctss/1971

DOI : $10.4000 /$ rdctss. 1971

ISSN : 2262-9815

Éditeur

Centre de droit comparé du travail et de la sécurité sociale

Édition imprimée

Date de publication : 1 avril 2018

Pagination : 6-17

ISSN : 2117-4350

\section{Référence électronique}

Vanessa De Greef, «Les formes variées et évolutives de l'activation en Belgique », Revue de droit comparé du travail et de la sécurité sociale [En ligne], 1 | 2018, mis en ligne le 01 novembre 2021, consulté le 13 novembre 2021. URL : http://journals.openedition.org/rdctss/1971 ; DOI : https:// doi.org/10.4000/rdctss. 1971

\section{(c) (i) (9)}

Revue de droit comparé du travail et de la sécurité sociale est mise à disposition selon les termes de la Licence Creative Commons Attribution - Pas d'Utilisation Commerciale - Pas de Modification 4.0 International. 


\section{LES FORMES VARIÉES ET ÉVOLUTIVES DE L'ACTIVATION EN BELGIQUE}

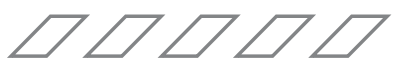

\section{RÉSUMÉ}

Cet article a pour but d'exposer de manière synthétique l'évolution des dispositifs d'activation dans le système de sécurité sociale belge, en faisant un bref récapitulatif historique et en insistant particulièrement sur les réformes des années 2016 et 2017.

MOTS CLÉS: Sécurité sociale, Activation, Belgique, Réinsertion professionnelle, Emploi.

\section{ABSTRACT}

The aim of this article is to summarize the evolution of activation mechanisms in the Belgian social security system, giving a brief historical summary and particularly emphasizing the reforms of the years 2016 and 2017.

KEYWORDS: Social security, Activation, Belgium, Professional reintegration, Employment. 


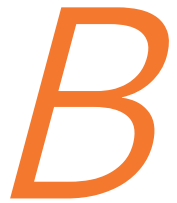

ien qu'on puisse retrouver en Belgique certains traits des mesures d'activation dès les années $70^{1}$, on constate, dès le début des années 90, différentes mesures dites expressément «actives » qui sont destinées aux chômeurs et qui se substituent aux programmes d'emploi. Ces mesures visent à mettre au travail différentes catégories de chômeurs au travers de statuts qui sont désormais à «mi-chemin » entre l'emploi et le chômage².

Toutefois, c'est véritablement en 1993 que la figure de l'État social dit «actif» devient plus visible lorsque, dans le domaine de l'aide sociale et que le phénomène de contractualisation de la protection sociale apparaît dans les rapports entre les centres publics d'action sociale (dits "CPAS", appelés jusqu'en 2004 centres publics d'aide sociale) et les bénéficiaires du revenu minimum (appelés depuis 2002, les bénéficiaires du droit à l'intégration sociale). Ainsi, le législateur belge emboîte le pas au législateur français ${ }^{3}$ - cinq années après précisément - en adoptant une loi qui marque l'avènement du contrat dont fait l'objet le « projet individualisé d'intégration sociale ». Depuis 2002, le droit à l'intégration sociale peut prendre la forme d'un emploi ou d'un revenu d'intégration, ces deux modalités pouvant être combinées et assorties chacune d'un contrat, appelé « projet individualisé $d^{\prime}$ intégration sociale $»^{4}$. Dans ce contrat, les parties fixent leurs engagements respectifs: le centre d'action sociale doit y «spécifier la nature de l'aide qu'il octroie à l'ayant droit, tandis que [ce dernier], de son côté, doit, en "contrepartie" de l'aide perçue, s'engager à accomplir un certain nombre de démarches destinées à faciliter son "intégration" sociale et professionnelle $\aleph^{5}$.

Si le droit à l'intégration sociale marque l'entrée de l'État social actif en Belgique, l'État belge n'a pas limité son action à ce champ de la protection sociale. Le gouvernement fédéral s'est ensuite attelé au domaine de l'assu rance chômage (I) avant de réformer ultérieurement, avec le législateur fédéral, le domaine de l'assurance indemnités (IV et V). Cependant, le droit à l'intégration sociale semble constituer une forme de laboratoire pour le législateur fédéral belge qui peut intervenir facilement dans ce domaine - sans devoir se concerter avec trop de niveaux de pouvoirs différents - et tester divers dispositifs d'activation, comme le dispositif de workfare (II) ou l'extension généralisée de l'outil contractuel entre les bénéficiaires et les centres publics d'action sociale (III). Nous présenterons ces évolutions de façon chronologique et analyserons la variété des traits des politiques dites d'activation.

1 D. Dumont, La responsabilisation des personnes sans emploi en question. Une étude critique de la contractualisation des prestations sociales en droit belge de l'assurance chômage et de l'aide sociale Bruxelles, La Charte, 2012, p. 425, $\mathrm{n}^{\circ} 727$.

2 Sur ce statut hybride, ibid., p. 291, n 534.

3 Voir R. Lafore, "Le contrat dans la protection sociale, une approche française », in Ph. Auvergnon (dir.) La contractualisation du droit social. Actes du séminaire international de droit comparé du travail, des relations professionnelles et de la sécurité sociale, Bordeaux, Comptrasec, 2003, p. 214.

4 Article 2 de la loi du 26 mai 2002 concernant le droit à l'intégration sociale, Moniteur belge, 31 juillet 2002.

5 D. Dumont, La responsabilisation des personnes sans emploi en question, op. cit., $\mathrm{n}^{\circ} 165, \mathrm{p} .100$. 


\section{I - L'INSTALLATION DE L'ÉTAT SOCIAL ACTIF AVEC L'ASSURANCE CHÔMAGE}

À partir des années 2000, la dynamique propreà unÉtat social « actif »va particulièrement s'accélérer dans le domaine de l'assurance chômage. Franck Vandenbroucke, socialiste flamand, et alors ministre de l'emploi, est inspiré par les idées d'Anthony Giddens et considère alors que "l'État social traditionnel fait défaut», étant donné que celui-ci "propose [aux assurés] une allocation, mais pas d'issue »; il ajoute que les «mécanismes de l'actuel système de sécurité sociale (...) découragent les gens au lieu de les encourager à être actifs ${ }^{6}$. Cette transformation de l'État social se remarque juridiquement avec la modification en 2004 d'une condition d'octroi de l'assurance chômage: celle de la disponibilité pour le marché de l'emploi. Jusqu'en 2004, cette condition d'octroi signifiait que le chômeur devait être inscrit comme demandeur d'emploi et être prêt à accepter tout emploi convenable. En 2004, le gouvernement fédéral belge réaffirme la condition de la disponibilité pour le marché de l'emploi en prévoyant que désormais, le chômeur doit être disponible passivement - ce qui correspondait à la définition réglementaire de la condition jusqu'en 2004 - mais également activement, c'est-à-dire qu'il doit rechercher activement un travail par lui-même ${ }^{7}$.

En 2012, le gouvernement fédéral décide de soumettre également à la condition de disponibilité pour le marché de l'emploi certains chômeurs qui étaient précédemment exemptés de la procédure, à savoir ceux qui présentent une capacité de travail réduite mais qui dépendent cependant de l'assurance chômage ${ }^{8}$. Depuis, diverses mesures ont été prises par le gouvernement fédéral qui lui a succédé pour introduire des exceptions dans la réforme adoptée et alléger cette activation " ordinaire », au vu des difficultés qu'elle soulevait?

Les réformes de 2004 et 2012 de l'assurance chômage semblent préfigurer « un retour au marché de la protection sociale » et un "abandon à l'assistance de ceux qui ne travaillent pas ou sont économiquement faibles ${ }^{10}$. En effet, l'écrémage des allocataires qui a lieu dans l'assurance chômage a eu pour effet d'augmenter massivement le nombre de bénéficiaires du droit à l'intégration sociale ${ }^{11}$. Ces derniers bénéficient généralement de

6 F. Vandenbroucke, cité par Geoffrey Geuens, "Les médias belges et l'État social actif. Genèse, reproduction et diffusion du nouveau sens commun socio-économique », Quaderni, $n^{\circ} 45$, Automne 2001, p. 22.

7 Arrêté royal du 4 juillet 2004 portant modification de la réglementation du chômage à l'égard des chômeurs complets qui doivent rechercher activement un emploi, Moniteur belge, 9 juillet 2004. Pour une analyse fouillée de cette réforme, voir D. Dumont, La responsabilisation des personnes sans emploi en question, op. cit., pp. 325-406.

8 Pour une analyse de cette réforme, voir V. De Greef, Droit au travail et troubles mentaux. Une analyse critique des exclusions et des inclusions par le droit en assurance chômage et en aide sociale, la Charte, coll. «Association belge pour le droit du travail et de la sécurité sociale », Bruxelles, 2016.

9 V. De Greef « L'activation silencieuse des personnes partiellement inaptes au travail dans l'assurance chômage ", Revue de droit social, 2016, n², pp. 243-275.

$10 \mathrm{Ph}$. Auvergnon, "L'hypothèse d'une contractualisation du droit social dans différents systèmes juridiques ", in Ph. Auvergnon (dir.) La contractualisation du droit social, Bordeaux, Comptrasec, 2003, p. 16.

11 Voir à ce sujet l'ouvrage de D. Zamora, De l'égalité à la pauvreté. Une socio-histoire de l'assistance en Belgique (1895-2015), éd. de l'Université de Bruxelles, Bruxelles, 2017, pp. 140-148. 
revenus beaucoup plus bas que dans l'assurance chômage ${ }^{12}$. Les bénéficiaires du droit à l'intégration sociale peuvent être aidés en vue de leur réinsertion professionnelle mais ils travaillent parfois aussi gratuitement dans le cadre d'un « service communautaire ».

\section{II - LE SERVICE COMMUNAUTAIRE : UNE DOUBLE PRÉCARISATION}

En 2016, un système de workfare a été adopté dans le domaine du droit à l'intégration sociale ${ }^{13}$. Il prévoit que la personne « peut » prester gratuitement un service communautaire pour démontrer sa disposition à travailler. Le cas échéant, le service communautaire est intégré au projet individualisé d'intégration sociale (PIIS ci-après).

Une circulaire du 12 octobre 2016 est ensuite venue préciser que «si la personne concernée ne souhaite plus effectuer le service, elle doit le signaler à son travailleur social et ils reverront ensemble les conditions du PIIS, en concertation ». II y a cependant fort à parier qu'il y aura un décalage entre les textes et la pratique. En effet, jusqu'à présent, le contenu du plan a été essentiellement déterminé par le travailleur social. En outre, le refus de signer ce plan peut donner lieu à un refus d'octroi du revenu d'intégration et le nonrespect du plan peut, quant à lui, aboutir à une suspension temporaire totale ou partielle du revenu d'intégration pendant un mois, ou trois mois en cas de 'récidive' dans l'année.

Deux recours sont actuellement pendants devant la Cour constitutionnelle et devant le Conseil d'État afin de vérifier la validité dans l'ordre juridique belge de la loi et de l'arrêté royal encadrant le service communautaire. Parmi les critiques avancées par les requérants, il y a entre autres l'atteinte potentielle à l'interdiction de travail forcé ${ }^{14}$, le fait que le service communautaire mis en place est un travail qui ne respecte ni les règles relatives aux contrats de travail, ni les règles qui s'appliquent aux prestations de travail effectuées sous l'autorité d'une autre personne ou encore le fait que cette loi ne fait pas de référence explicite à la loi du 3 juillet 2005 relative aux droits des volontaires ${ }^{15}$.

12 Bien que l'écart entre le montant des allocations de chômage et celui du revenu d'intégration se réduise de plus en plus suite à la réforme de la dégressivité renforcée des allocations de chômage adoptée durant l'été 2012. Voir D. Dumont, «Dégressivité accrue des allocations de chômage versus principe de standstill ", Journal des tribunaux, n 6541, 2013/39, pp. 769-776; P. Palsterman, "Les réformes de l'été en matière de chômage », in F. Etienne et M. Dumont (dir.) Regards croisés sur la sécurité sociale, Anthemis, coll. Commission université-palais, Liège, 2012, pp. 952 à 966.

13 Voir la loi du 21 juillet 2016 modifiant la loi du 26 mai 2002 concernant le droit à l'intégration sociale (Moniteur belge, 2 août 2016), l'arrêté royal du 3 octobre 2016 modifiant l'arrêté royal du 11 juillet 2002 portant règlement général en matière de droit à l'intégration sociale, (Moniteur belge, 11 octobre 2016) et la circulaire du 12 octobre 2016 relative à la loi du 21 juillet 2016 modifiant la loi du 26 mai 2002 concernant le droit à l'intégration sociale (non publiée au Moniteur belge).

14 E. Dermine, "Activation Policies for the Unemployed and The International Case Law on the Prohibition of Forced Labour ", Journal européen des droits de I'homme/European Journal of Human Rights, vol. 1, n5, 2013, pp. 746-776; E. Dermine et V. De Greef, « Le droit au travail librement entrepris (art. 1er, $\S 2$ de la CSE) face aux situations de travail non protégées par le droit social. Les cas du travail pénitentiaire et des mesures de workfare ", in S. Van Drooghenbroeck, F. Dorssemont et G. Van Limberghen (dir.), Actualités des droits sociaux fondamentaux, Bruxelles, La Charte, 2016, pp. 315 à 317.

15 Moniteur belge, 29 août 2005. 
Cette transformation dans le droit à l'intégration sociale belge dessine ce que d'aucuns nomment une dualisation naissante au sein de l'État social entre des insiders globalement bien protégés et la marge croissante des outsiders précaires et inactifs ${ }^{16}$. Ce déplacement, qui se fait parallèlement en France, pourrait confronter les allocataires à "une double peine » en étant «mis à l'écart à la fois du travail et de ses protections ${ }^{17}$. En plus d'une précarisation du droit du travail des outsiders, le dispositif pourrait retentir sur le taux d'emploi. Ainsi, «plus le workfare comportera une contribution à la production de biens et de services, plus grand est le risque qu'il se substitue à de l'emploi standard ${ }^{18}$. Malgré la volonté du ministre de l'intégration sociale que ce ne soit pas le cas, rien n'exclut cette tendance en raison de la définition particulièrement élusive du « service communautaire».

Un tel dispositif pourrait pénétrer le domaine de l'assurance chômage. Ainsi, l'accord du gouvernement fédéral belge pour les années 2014-2019 prévoit que le chômeur devrait à l'avenir travailler gratuitement dans le cadre d'un service dit « communautaire $»^{19}$. Dans le domaine de l'assurance chômage, c'est la notion d'emploi convenable - qui est notamment le garant d'un emploi de qualité - qui se verrait remise en cause. Comme l'explique l'économiste B. Van der Linden, ce système représenterait « une rupture puisqu'il ne s'agit pas d'offrir un emploi convenable mais une occupation [non rémunérée] dans des "services à la communaute" $»^{20}$.

\section{III - L'ÉTAT SOCIAL ACTIF POUR TOUS LES BÉNÉFICIAIRES DU DROIT À L'INTÉGRATION SOCIALE?}

Outre le service communautaire, une autre réforme importante a eu lieu dans le domaine du droit à l'intégration sociale en 2016. Alors que le projet individualisé d'intégration sociale (PIIS) n'était obligatoire que pour les jeunes de 18 à 25 ans et qu'il n'était conclu qu'en vue d'obtenir un contrat de travail, de suivre une formation ou d'obtenir un diplôme, le législateur prévoit la généralisation de cet outil à tous les bénéficiaires du droit à l'intégration sociale des CPAS - sauf quelques rares exceptions relatives à des situations d'équité ou de santé - et adapte les subsides accordés aux CPAS en fonction de ce surcroît de travail.

Dans une étude commanditée par le service public de programmation (SPP) Intégration sociale, à la demande du Ministre fédéral de l'intégration sociale, parue en 2015 - avant

16 P. Taylor-Gooby, "New Social Risks and Welfare States: New Paradigm and New Politics? », in P. Taylor-Gooby (dir.), New risks, New Welfare. The transformation of the European Welfare State, Oxford university press, Oxford, 2004, p. 225. N. Van Mechelen et D. Zamora, " Les reconfigurations de l'assistance et de la sécurité sociale en Belgique (1925-2015) », in I. Pannecoucke (dir.), Annuaire de la pauvreté en Belgique, ACCO, Leuven, 2016, p. 127.

17 N. Duvoux, Le nouvel âge de la solidarité. Pauvreté, précarité et politiques publiques, La république des idées, Seuil, Paris, 2012. V. De Greef et D. Zamora, "Le système de sécurité sociale belge: de I'universalisation à la dualisation », in I. Daugareilh et M. Badel (dir.), La sécurité sociale: 70 ans d'âge, une idée neuve, éd. Pedone, 2018, à paraître.

18 B.Van Der Linden, "Chômage indemnisé contre service à la communauté? ", Regardséconomiques, septembre 2014, n 114, disponible sur le site de la revue, http://www.regards-economiques.be/ images/reco-pdf/reco 144.pdf

19 Doc. Parl., Chambre, Déclaration de politique gouvernementale, 14 octobre 2014, n0020/001, p. 28-29.

20 B. Van Der Linden, « Chômage indemnisé contre service à la communauté? », op.cit. 
l'adoption de la réforme donc -, des chercheurs de l'Université Saint Louis et de Karel De Grote Hogeschool mettaient en évidence que:

"Le scénario d'une généralisation du PIIS à tous les usagers peut être vertueux, mais il comporte en effet également plusieurs risques. Le premier risque est que la généralisation du PIIS à tous les usagers, loin de favoriser un accompagnement personnalisé, conduise à un traitement standardisé et bureaucratique. Certes, tous les usagers signeraient un "PIIS", mais l'adéquation de ce PIIS à leurs besoins d'intégration sociale serait purement formelle. Dans beaucoup de CPAS, compte tenu de l'insuffisance des moyens et des ressources d'intégration sociale que les CPAS et leurs partenaires peuvent effectivement proposer aux usagers, cela conduirait à établir des PIIS de manière purement formelle et fictive ou à proposer des PIIS en inadéquation avec la situation et les besoins des usagers et les possibilités du contexte.

Le risque de la généralisation du PIIS, dans son format actuel et compte tenu des divergences de philosophie, de politique, et de pratiques des 589 CPAS de Belgique, est que cette généralisation ne soit, dans certains cas, une porte ouverte à des pratiques arbitraires et discrétionnaires des CPAS envers les usagers, au gré des impulsions politiques locales. Alors que I'histoire des CPAS témoigne du passage de l'arbitraire d'une assistance attribuée au cas par cas (comme cela était le cas à l'époque des CAP - Commissions d'Assistance Publique - qui ont précédé les CPAS entre 1925 et 1975) à la reconnaissance de droits sociaux (art. 1er. de la loi organique des centres publics d'action sociale 8 juillet 1976: 'Toute personne a droit à l'aide sociale. Celle-ci a pour but de permettre à chacun de mener une vie conforme à la dignité humaine'), le risque d'une généralisation du PIIS est bien de réintroduire une appréciation locale et subjective du droit à l'intégration sociale, renforçant encore davantage une inéquité dans les conditions d'octroi et de maintien du RIS. Alors que les évolutions des situations sociales et les transformations des politiques en matière de sécurité sociale (avec les restrictions apportées au bénéfice des allocations de chômage pour un nombre croissant de personnes (fin de droit, dégressivité, conditions d'accès et de maintien à l'allocation d'insertion) ont conduit à augmenter de manière importante le nombre de demandeurs d'aide sociale auprès des CPAS, une généralisation d'une particularisation, via le PIIS, des conditions d'octroi et de maintien du RIS risque de se transformer, dans certains cas, en instrument de précarisation et d'exclusion de ce qui constitue dans notre système social le "dernier filet". Même si la très grande majorité des CPAS et des travailleurs sociaux travaillent dans une perspective d'intégration sociale, l'enquête menée auprès des CPAS montre qu'il convient également de prévenir le risque que le PIIS ne soit utilisé comme instrument d'exclusion sociale ${ }^{21}$.

Un des recours pendants devant la Cour constitutionnelle - le même que celui introduit au sujet du service communautaire - doit permettre de vérifier la constitutionnalité de la généralisation du projet individualisé d'intégration sociale (PIIS) dans l'ordre juridique belge. Une des critiques émises dans la requête concerne le fait que le législateur a fait le

21 A. Franssen, L. Méhauden, K. Driessens et J. Depauw, Le projet individualisé d'intégration sociale. Recherche évaluative et prospective au sein des CPAS belges, Rapport de recherche 2015, pp. 9495. : http://hdl.handle.net/2078.3/173117 
choix délibéré de ne plus encadrer la manière dont l'intégration professionnelle ou sociale doit être réalisée. Cela aurait pour effet, est-il précisé, que:

"Chacun dans le Royaume a son opinion personnelle concernant les facteurs qui facilitent ou entravent "l'intégration professionnelle" ou "l'intégration sociale" et concernant les attitudes qui vont ou non renforcer les chances de décrocher un emploi ou de bénéficier de cette intégration». II en découle que "dans un tel contexte, les notions d' "intégration professionnelle" et d' "intégration sociale" apparaissent comme beaucoup trop larges et beaucoup trop dépendantes des interprétations subjectives qui peuvent en être faites pour constituer un quelconque encadrement des obligations qui ont été imposées dans les PIIS ».

Le fait que le législateur confie au pouvoir exécutif le soin de régler des aspects déterminants du droit à l'aide sociale et du droit au respect de la vie privée pourrait heurter le principe de légalité qui requiert une habilitation suffisamment précise au Roi ${ }^{22}$.

\section{IV - L'ACTIVATION VOLONTAIRE: L'EXEMPLE DE L'ASSURANCE INVALIDITÉ}

Quant à l'assurance invalidité, destinée aux personnes en incapacité de travail, cela fait seulement, comparativement aux autres branches de la sécurité sociale, une dizaine d'années que les autorités publiques commencent à s'intéresser aux programmes de réinsertion professionnelle dans ce domaine ${ }^{23}$. En 2006, une nouvelle philosophie a été adoptée dans cette branche de la sécurité sociale et a modifié fondamentalement, en 2009, le rôle du médecin-conseil. Celui-ci ne va plus simplement juger de l'indemnisation de l'assuré en évaluant son incapacité de travail mais il va également préparer le processus de réadaptation professionnelle de l'assuré ${ }^{24}$. C'est également en 2009 que des prises en charge individuelles commencent à être mises en place pour les assurés. À partir de la fin de l'année 2010, l'optique poursuivie par l'Institut national de l'assurance maladie invalidité (INAMI), l'administration en charge de l'assurance soins de santé et indemnités, est de se rapprocher des organismes régionaux et communautaires en charge de l'insertion et de la formation professionnelles afin d'organiser la prise en charge professionnelle des assurés qui reçoivent une allocation d'invalidité. À cette fin, des conventions sont conclues entre I'Institut national, les mutuelles et lesdits organismes.

22 D. Dumont, «Que reste-t-il du principe de légalité en droit de la sécurité sociale? Sécurité sociale et démocratie parlementaire », $n^{\circ}$ spécial «Aux sources du droit social. En hommage à Micheline Jamoulle/Sociaal recht, over bronnen en herbronnen. Als eerbetoon aan Micheline Jamoulle », sous la dir. de D. Dumont et F. Dorssemont (dir.), Revue de droit social/Tijdschrift voor Sociaal Recht, 2017, n 1-2, pp. 113-146.

23 Voir la loi du 13 juillet 2006 portant dispositions diverses en matière de maladies professionnelles, d'accidents du travail et en matière de réinsertion professionnelle (Moniteur belge, $1^{\text {er }}$ septembre 2006) ainsi que l'arrêté royal du 3 juillet 1996 portant exécution de la loi relative à l'assurance obligatoire soins de santé et indemnités coordonnée le 14 juillet 1994 (Moniteur belge, 31 juillet 1996).

24 Articles 109 bis et $153 \S 2$ de la loi coordonnée du 14 juillet 1994 relative à l'assurance soins de santé et indemnités (Moniteur belge, 27 août 1994). 
La grande différence entre l'assurance soins de santé et indemnités-invalidité, d'une part, et l'assurance chômage et l'aide sociale, d'autre part, est que le bénéfice d'un programme de réadaptation professionnelle ou la reprise du travail est volontaire, dans la mesure où le consentement de l'assuré est requis pour déclencher ce type de mesures et que la cessation ou le refus de bénéficier d'un tel programme ou de reprendre le travail n'a aucun impact sur le versement des allocations ${ }^{25}$. Pour évaluer le caractère volontaire, il importe de vérifier si la personne peut «exprimer sa volonté ${ }^{26}$. II est indispensable d'observer si elle comprend la nature d'un programme de réinsertion professionnelle ainsi que les conséquences de son accord à participer à ce programme. Il faut, en outre, que la communication avec la personne se déroule dans une langue claire ${ }^{27}$.

II faudra cependant suivre les évolutions législatives car le gouvernement fédéral souhaite introduire, à l'avenir, des sanctions pour les assurés qui ne se présentent pas chez le médecin conseil. En mars 2017, le gouvernement fédéral a annoncé son intention d'introduire des sanctions en cas de manquement des assurés aux obligations administratives ou de non-présentation à un entretien avec le médecin-conseil. Ces assurés étant en incapacité de travail, le dossier est particulièrement sensible et de telles sanctions pourraient aboutir à des situations dramatiques et incohérentes avec leur situation médicale. Plusieurs des acteurs consultés - dont le Conseil National du Travail - se sont positionnés contre la mise en place de telles sanctions.

La question du caractère volontaire de l'accompagnement n'est évidemment pas l'unique question qui doit se poser à nous. Si l'analyse "sous l'angle de la contrainte à la reprise d'un emploi (le devoir de travailler)» nous semble capitale, celle « de l'augmentation des capacités de trouver un emploi de qualité (la liberté positive de travailler) » nous semble tout aussi essentielle et encore fréquemment négligée dans les pays de l'OCDE ${ }^{28}$. Les deux sont cependant plus liées qu'il n'y paraît: I'OIT a en effet reconnu que des conditions de travail très pénibles - liées à un emploi de qualité - peuvent révéler une atteinte au libre choix de l'emploi et, dans les cas les plus graves, une situation de travail forcéé.

25 Ceci a été précisé en 2011 par le législateur lorsqu'il a modifié la loi du 14 juillet 1994 relative à l'assurance soins de santé et indemnités-invalidité pour supprimer le caractère préalable de l'autorisation de reprise du travail et la remplacer par une autorisation qui peut être octroyée a posteriori (Projet de loi-programme, Exposé des motifs, Doc. Parl., Chambre, 19 mai 2011, $\left.\mathrm{n}^{\circ} 1481 / 001\right)$.

26 Art. 14 de la loi du 22 août 2002 relative aux droits du patient, Moniteur belge, 26 septembre 2002.

27 Art.7, §2 de la loi du 22 août 2002 relative aux droits du patient, Moniteur belge, 26 septembre 2002.

28 J.-M. Bonvin et E. Maochon, «Droit au travail et responsabilité individuelle dans les États sociaux contemporains. Une analyse en termes de capabilités des politiques d'activation des personnes sans emploi » in E. Dermine et D. Dumont (dir.), Activation Policies for the Unemployed, the Right to Work and the Duty to Work, Bruxelles, P.I.E. Peter Lang, pp. 779 et 802.

29 OIT, Rapport du Directeur général du BIT, Une alliance mondiale contre le travail forcé, Rapport global en vertu du suivi de la Déclaration de I'OIT relative aux principes et droits fondamentaux au travail, Rapport I(B), CIT, 93e session, Genève, 2005, p. 9, § 31 et p. 70, § 295. Voir E. Dermine et V. De Greef, «Le droit au travail librement entrepris (art. 1er, § 2 de la CSE) face aux situations de travail non protégées par le droit social. Les cas du travail pénitentiaire et des mesures de workfare», op. cit., p. 318. 
Il en découle que si les conditions de travail ne sont pas conformes au droit fondamental à des conditions de travail équitables, le consentement donné par le travailleur peut être invalidé ${ }^{30}$.

\section{V - LA SÉCURISATION DE LA RELATION DE TRAVAIL, UN OBJECTIF COLLATÉRAL DE L'ACTIVATION DANS L'ASSURANCE INVALIDITÉ-EN ESPĖCES?}

Avant de revenir éventuellement avec un nouveau projet de texte relatif aux sanctions, le gouvernement fédéral a déjà introduit de nouveaux trajets de réinsertion pour les assurés en incapacité de travail et a réformé également le droit du travail en vue d'encadrer la relation de travail entre les travailleurs ayant une incapacité de travail et leurs employeurs ${ }^{31}$. C'est par le biais d'une loi-programme du 19 décembre 2014 - appelée souvent loi « fourretout» en raison du nombre et de la diversité des matières traitées - que la loi relative à l'assurance soins de santé et indemnités, coordonnée le 14 juillet 1994, a été modifiée. Elle prévoit que, au plus tard trois mois après le début de la période d'incapacité primaire et après une consultation approfondie avec le médecin-conseil et tous les acteurs à impliquer, " un plan de réintégration multidisciplinaire est établi à l'intention du titulaire pour lequel une réintégration peut être envisagée au vu de ses capacités restantes ${ }^{32}$. Un tel plan était déjà mis en place dans le passé mais pas de façon systématique, ni organisé à une telle cadence.

En droit du travail, il a fallu attendre deux ans - au cours desquels divers groupes de travail se sont réunis - pour que le législateur intervienne. L'exposé des motifs de la loi du 20 décembre 2016 prévoit que la réforme vise à « sécuriser juridiquement la relation

30 Cela concerne la jurisprudence générale relative à l'interdiction du travail forcé développée par l'Organisation internationale du travail, par la Cour européenne des droits de l'homme et par le Comité européen des droits sociaux. Voir E. Dermine et V. De Greef, « Le droit au travail librement entrepris (art. 1er, § 2 de la CSE) face aux situations de travail non protégées par le droit social. Les cas du travail pénitentiaire et des mesures de workfare », op. cit. et pour une analyse plus approfondie, E. Dermine, Droit au travail et politiques d'activation des personnes sans emploi. Étude critique du rôle du droit international des droits humains, Bruxelles, Bruylant, 2017.

31 La réforme a donné lieu à cinq textes: la loi-programme du 19 décembre 2014 (Moniteur belge, 29 décembre 2014), l'arrêté royal du 28 octobre 2016 modifiant l'arrêté royal du 28 mai 2003 relatif à la surveillance de la santé des travailleurs en ce qui concerne la réintégration des travailleurs en incapacité de travail (Moniteur belge, 1er décembre 2016), l'arrêté royal du 8 novembre 2016 modifiant l'arrêté royal du 3 juillet 1996 portant exécution de la loi relative à l'assurance obligatoire soins de santé et indemnités-invalidité coordonnée le 14 juillet 1994 en ce qui concerne la réinsertion socioprofessionnelle (Moniteur belge, $1^{\text {er }}$ décembre 2016), la loi du 20 décembre 2016 portant dispositions diverses en droit du travail liées à l'incapacité de travail (Moniteur belge, 9 janvier 2017) et l'arrêté royal du 30 janvier 2017 modifiant l'arrêté royal du 28 mai 2003 relatif à la surveillance de la santé des travailleurs (Moniteur belge, 6 février 2017). Sur ces réformes, voir A. Mortier, "La réforme tendant à faciliter la réintégration des travailleurs en incapacité de travail ", Revue de la Faculté de droit de I'Université de Liège, 2017/2, pp. 357-378; M. Vanhegen, Arbeidsongeschiktheid en re-integratie in het arbeidsrecht, Bruges, La Charte, 2017; D. Caccamisi, «Le nouveau trajet de réintégration des travailleurs en incapacité de travail: un aperçu de la réforme et de ses conséquences sur la force majeure médicale », Orientations, 2018, n 1, pp. 2-27.

32 Moniteur belge, 29 décembre 2014. Cette loi-programme insère à l'article 100 de la loi du 14 juillet 1994, un paragraphe $1 / 1$. 
de travail durant la période de travail adapté $»^{33}$. Tout d'abord, il est désormais prévu que l'exécution du contrat de travail initial n'est pas suspendue lorsque le travailleur a été autorisé par le médecin-conseil à reprendre temporairement le travail auprès de son employeur et que ce dernier est d'accord. Il existe, dans ce cas, une présomption réfragable de maintien de la relation de travail initiale. Comme par le passé, un système de cumul de l'allocation et d'une partie du salaire est mis en place. Ensuite, une nouvelle réglementation est prévue en cas d'incapacité de travail temporaire ou définitive du travailleur et vise à enclencher un trajet de réintégration pour éviter la rupture du contrat, sauf lorsque le travailleur «est définitivement inapte à reprendre le travail convenu et n'est en état d'effectuer chez l'employeur aucun travail adapté ni un autre travail ${ }^{34}$. Ce faisant, cette réforme est venue clôturer une longue controverse jurisprudentielle et doctrinale au sujet du moment où l'employeur pouvait rompre un contrat de travail en cas de force majeure ou d'impossibilité définitive du travailleur d'exercer le travail convenu. Désormais, une telle rupture ne peut avoir lieu avant que le trajet de réintégration ne soit définitivement achevé. En raison de l'instauration de cette procédure, cette réforme devrait aller à l'encontre de la jurisprudence majoritaire de l'époque qui «revenait de facto à tenir en échec toute politique de réintégration des travailleurs malades $»^{35}$.

Cela dit, après un premier trimestre d'application de la réforme en 2017, l'innovation majeure annoncée pose question. La Fédération générale du travail de Belgique fédération syndicale à tendance socialiste - constate que dans $72 \%$ des décisions des médecins du travail, ceux-ci déclarent les travailleurs inaptes à reprendre le travail, ce qui a pour conséquence de rompre le contrat de travail pour force majeure médicale ${ }^{36}$. Ces chiffres devront être confirmés pour les prochains trimestres et certains acteurs de terrain critiquent leur fiabilité. Si le pourcentage annoncé par la FGTB devait être validé par la suite, on s'éloignerait du but du législateur qui était de « sécuriser la relation de travail ».

\section{Pour conclure (provisoirement) sur les formes multiples et variées de l'activation}

On assiste ainsi à un développement de l'activation des allocataires sociaux dans les différents secteurs de la sécurité sociale. Cette activation a des modalités très diverses: par exemple, la nature de l'activation peut être contrainte ou volontaire. Le niveau de contrainte peut varier de degrés faibles allant d'une invitation à prester du service

33 Projet de loi portant dispositions diverses en droit du travail liées à l'incapacité de travail, Exposé des motifs, Doc. Parl., Chambre, 16 novembre 2016, n 54-2155/001, p. 3.

34 Nouvel art. 73/2, §4 de l'arrêté royal du 28 mai 2003 relatif à la surveillance de la santé des travailleurs en ce qui concerne la réintégration des travailleurs en incapacité de travail suite à l'arrêté royal du 28 octobre 2016 modifiant l'arrêté royal du 28 mai 2003 relatif à la surveillance de la santé des travailleurs en ce qui concerne la réintégration des travailleurs en incapacité de travail (Moniteur belge, $1^{\text {er }}$ décembre 2016).

35 D. Caccamisi, «Le nouveau trajet de réintégration des travailleurs en incapacité de travail: un aperçu de la réforme et de ses conséquences sur la force majeure médicale », op. cit., p. 6.

$36 \mathrm{FGTB}$, "Le trajet de réintégration des malades, une machine à licenciements pour les employeurs », 27 avril 2017

http://www.fgtb.be/-/le-trajet-de-reintegration-des-malades-une-machine-a-licenciements-pourles-employeurs, consulté le 26 septembre 2017. 
communautaire à des degrés plus élevés, tels qu'une obligation de rechercher activement un emploi convenable. L'activation peut viser la réinsertion professionnelle ou la réinsertion sociale. Elle peut favoriser la conclusion d'un contrat de travail, le recours au bénévolat ou désormais, au service communautaire. Elle peut avoir pour effet de responsabiliser l'assuré social ou de renforcer les obligations des institutions de sécurité sociale, et, parfois, les deux concomitamment. L'activation peut encore se déployer sous différentes formes en fonction de l'accompagnement prodigué. Il peut s'agir d'un accompagnement soutenu, très balisé ou d'un encadrement plus léger, voire inexistant. II pourrait être intéressant d'évaluer, dans chaque secteur de la sécurité sociale, ne fût-ce que le temps accordé par chaque conseiller à l'assuré pour l'aider dans sa recherche d'emploi, de formation ou d'activité.

L'application des règles peut, elle aussi, donner lieu à une activation différenciée dans le pays. Ainsi, il est possible que dans le domaine de l'assurance indemnités, le caractère volontaire d'un programme connaisse différents degrés ( $d$ 'un consentement éclairé à un consentement implicite par exemple). Dans le domaine du droit à l'intégration sociale, des travailleurs sociaux souhaitent que le service communautaire reste volontaire en décidant qu'il ne peut être utilisé pour démontrer sa disposition à travailler (et que la personne doit être exemptée de démontrer cette condition d'octroi). Certains CPAS ont été un pas plus loin et ont décidé de "boycotter» le service communautaire, en ne proposant tout simplement pas aux allocataires de recourir à un tel dispositif.

À l'heure actuelle, l'activation a donc des modes opératoires très divers. On peut regretter que face à un tel laboratoire de politiques sociales, les évaluations des politiques d'activation demeurent relativement rares. Si l'activation, peu importent ses contours, favorisait systématiquement la réinsertion sur le marché du travail en permettant à la personne d'avoir accès à un emploi de qualité, correspondant à ses qualifications, beaucoup seraient demandeurs d'une activation généralisée. Or, il apparaît que les effets des politiques d'activation mises en place ne sont pas si clairs ${ }^{37}$. En Belgique, le rapport 2014 du Conseil supérieur de l'emploi enjoint certes de poursuivre les politiques d'activation mais précise également que «l'efficacité en termes de retour à l'emploi de ces procédures et des sanctions éventuelles auxquelles elles peuvent donner lieu est difficile à évaluer [...]. L'impact d'une sanction sur le retour à l'emploi n'a de fait pas pu être démontré de manière univoque $»^{38}$. En France, parmi les études qui ont examiné les politiques d'activation, citons celle de 2012 réalisée sur des jeunes chômeurs français qui révèle que les politiques d'activation mises en place en France peuvent avoir pour effet d'augmenter le taux d'emploi des chômeurs accompagnés, mais qu'elles réduisent simultanément la chance des allocataires non accompagnés de trouver un poste de travail ${ }^{39}$. Autrement dit, les politiques d'activation ont pour effet de changer l'ordre dans la file d'attente pour l'emploi sans augmenter le niveau d'emploi total.

37 Des résultats positifs mais limités ont surtout pu être montrés à l'égard des jeunes peu scolarisés dans la mesure où il y avait une offre d'emploi suffisamment abondante. B. Cockx, M. Dejemeppe et B. Van der Linden, "L'activation du comportement de recherche favorise-t-elle un retour plus rapide à l'emploi? », Regards économiques, n 85, février 2011, 16 p.

38 Conseil supérieur de l'emploi, rapport 2014, juin 2014, p. 10.

39 B. Crepon, E. Duflo, M. Gurgand, R. Rathelot et P. Zamora, « Do Labor Market Policies have Displacement Effects? Evidence from a Clustered Randomized Experiment », The Quarterly Journal of Economics, vol. 128, n², 2013, pp. 531-580. 
Au-delà du souhait d'une évaluation plus systématique des politiques publiques, certains domaines pourraient être davantage coordonnés. On pense en particulier au manque de cohérence entre les branches de la sécurité sociale, notamment en ce qui concerne l'évaluation de la capacité de travail et l'accompagnement prodigué. En Belgique, le système de protection sociale de la personne souffrant d'une inaptitude au travail est particulièrement " éclaté ${ }^{40}$. Dans le meilleur des cas, cette personne est amenée à relever d'un secteur de la protection sociale contributif tel que l'assurance chômage ou l'assurance soins de santé et indemnités. À défaut, elle peut recourir, si du moins elle se trouve dans un «état de besoin » sur le plan pécuniaire, à l'allocation de remplacement de revenus destinée aux personnes handicapées, ou encore, en cas de refus de cette demande, à l'aide sociale de dernière ligne allouée par les CPAS (droit à l'intégration sociale et aide sociale au sens strict). Ces allers et retours entre régimes assurantiels et assistantiels démontrent la nécessité d'une réflexion plus approfondie sur le profil des assurés qui dépendent des allocations de chômage ou de l'assurance soins de santé et indemnités. Les personnes qui ne sont plus réellement aptes à travailler, doivent-elles continuer à relever de l'assurance chômage?

Après des décennies d'inaction, le monde politique semble aujourd'hui enfin disposé à mener ce débat puisqu'un projet est actuellement à l'étude afin d'éviter les situations de ballotement entre institutions de sécurité sociale. Le 25 avril 2014, un Collège national de médecine d'assurance sociale en matière d'incapacité de travail a été créé, notamment pour « proposer des méthodes standardisées d'évaluation de l'incapacité de travail dans le but d'une harmonisation des évaluations dans les différentes branches de la sécurité sociale ${ }^{41}$. Un tel Collège parviendra-t-il à dépasser les exigences budgétaires et les réflexes propres à chaque branche? Ses membres pourront-ils penser la sécurité sociale comme un tout qui permet d'évaluer de façon cohérente les capacités et les incapacités de travail? Cette réflexion aura-t-elle lieu en vue de donner aux allocataires sociaux un revenu et un accompagnement digne, peu importe la branche de sécurité sociale concernée? C'est à espérer.

40 Voir P. Palsterman, « L'incapacité de travail des travailleurs salariés dans le droit belge de la sécurité sociale: approche transversale », Chroniques de droit social, 2004, n 6, p. 305.

41 Art. 16 et 17 de la loi du 25 avril 2014 portant des dispositions diverses en matière de sécurité sociale, Moniteur belge, 6 juin 2014.

\section{VANESSA DE GREEF}

Chercheuse et maître d'enseignement à l'Université libre de Bruxelles. Thèmes de recherche: droit social, santé mentale et droit, politiques institutionnelles, droits fondamentaux, idéologie du droit.

\section{Publications:}

$\sim$ V. De Greef, « Les constructions et fondements idéologiques multiples du droit au travail des chômeurs souffrant de troubles mentaux en Belgique ", Administration Publique, 2017, $\mathrm{n}^{\circ} 3$, pp. 188-201.

V. De Greef et J. Porta, « Commentaire de I'article 32. Interdiction du travail des enfants et protection des jeunes au travail ", Charte des droits fondamentaux de I'Union européenne, Commentaires article par article, sous la dir. de F. Picod et S. Van Drooghenbroeck avec la collaboration de C. Rizcallah, Bruxelles, Larcier, 2018, pp. 695-712. 